\title{
Exotic states of matter with polariton chains
}

\author{
Kirill P. Kalinin, ${ }^{1,2}$ Pavlos G. Lagoudakis, ${ }^{1,3}$ and Natalia G. Berloff ${ }^{1,2, *}$ \\ ${ }^{1}$ Skolkovo Institute of Science and Technology Novaya Street, 100, Skolkovo 143025, Russian Federation \\ ${ }^{2}$ Department of Applied Mathematics and Theoretical Physics, University of Cambridge, Cambridge CB3 OWA, United Kingdom \\ ${ }^{3}$ Department of Physics and Astronomy, University of Southampton, Southampton, SO17 1BJ, United Kingdom
}

(Received 24 April 2017; revised manuscript received 5 October 2017; published 4 April 2018)

\begin{abstract}
We consider linear periodic chains of exciton-polariton condensates formed by pumping polaritons nonresonantly into a linear network. To the leading order such a sequence of condensates establishes relative phases as to minimize a classical one-dimensional $X Y$ Hamiltonian with nearest and next-to-nearest neighbors. We show that the low-energy states of polaritonic linear chains demonstrate various classical regimes: ferromagnetic, antiferromagnetic, and frustrated spiral phases where quantum or thermal fluctuations are expected to give rise to a spin-liquid state. At the same time nonlinear interactions at higher pumping intensities bring about phase chaos and novel exotic phases.
\end{abstract}

DOI: 10.1103/PhysRevB.97.161101

Frustrated spin systems represent one of the most demanding problems of condensed-matter physics $[1,2]$. Various strongly correlated states are realized by Hubbard models $[3,4]$ that, in particular limits, reduce to spin models. Geometric frustration leads to a rich variety of possible spin configurations in the ground and excited states of these systems due to the competition between interactions and the geometry of the spin lattice with a potential of creating novel, exotic classical, and quantum phases, such as resonating valence-bond states and valence-bond solids, as well as various kinds of quantum, topological, and critical spin liquids [5,6]. A spin liquid is a much sought state of matter with applications to high-temperature superconductivity and quantum computation. In this state, spins fluctuate in a liquid form without ever solidifying even in the ground state [2]. In real systems, the classical spin Heisenberg Hamiltonians are at best approximate. The real Hamiltonians are affected by assorted perturbations, such as quantum and thermal fluctuations, anisotropies, disorder, dipolar interactions, and coupling to lattice degrees of freedom. Depending on interactions and connectivity classical Heisenberg models may exhibit frustration with a large ground-state degeneracy. The ground-state manifold has no energy scale of its own, and therefore such perturbations may bring about unusual disordered spin liquids, Bose metal states with exotic excitations, and novel phase transitions. The theoretical possibility of quantum fluctuations combined with geometric spin frustration has been hotly debated since Anderson proposed them in 1973 [7] and only recently have been realized experimentally [8]. It remains to be seen which exotic phases other types of perturbations can produce. This can be elucidated by studying emergence phenomena: the dynamical generation of new types of degrees of freedom using some condensed-matter systems to mimic (simulate) spontaneously and collectively different ones, possibly unknown or otherwise unrealizable.

\footnotetext{
${ }^{*}$ Corresponding author: n.g.berloff@damtp.cam.ac.uk
}

Various systems have been proposed to act as analog classical or quantum simulators to mimic condensed-matter phenomena and realize disparate kinds of spin models: ultracold atomic and molecular gases in optical lattices [9-12], defects and vacancies in semiconductors or dielectric materials [13,14], magnetic impurities embedded in solid helium [15], photons [16], trapped ions [17,18], superconducting qubits [19], a network of optical parametric oscillators [20,21], and coupled lasers [22].

Recently, we introduced polariton graphs as a new platform to study unconventional superfluids, spin liquids, and many other systems based on the $X Y$ Hamiltonian [23]. Polariton condensates can be imprinted into any two-dimensional graph by spatial modulation of the pumping laser [24] and can be easily scalable to a large number of lattice sites. In the regime of nonresonant excitation, the individual condensates in a polariton graph select their relative phases without the influence of the pumping laser. The condensation is driven by bosonic stimulation so that a polariton graph condenses at the state with the phase configuration that carries the highest polariton occupancy [25], which corresponds to the global minimum of the $X Y$ Hamiltonian. The structure of the $X Y$ Hamiltonian is set by the interaction strengths among the condensates that are defined by the system's parameters, such as the graph geometry, the pumping profile, and intensity. The $X Y$ model is a mathematical abstraction of the spin system, such as a disordered magnetic material composed of competitively interacting spins. For such a system, a spin indexed by $i$ is represented by a two-dimensional unit vector $\mathbf{s}_{i}=\left(\cos \theta_{i}, \sin \theta_{i}\right)$, and the energy is expressed by the $X Y$ Hamiltonian $H_{X Y}=-\sum_{i<j} J_{i j} \mathbf{s}_{i} \cdot \mathbf{s}_{j}=-\sum_{i<j} J_{i j} \cos \left(\theta_{i}-\right.$ $\theta_{j}$ ), where $J_{i j}$ denotes the coupling coefficient between the $i$ th and $j$ th spins. We refer to the coupling as ferromagnetic (antiferromagnetic) if $J_{i j}>0\left(J_{i j}<0\right)$. The $X Y$ model is a limiting case of the Heisenberg spin model or $n$-vector model for $n=2$. The vectors correspond to the directions of the spins (originally quantum mechanical) in a material in which the $z$ component of the spins couples less than the 
$x$ and $y$ components. Since $H_{X Y}$ is the simplest model that undergoes the $U(1)$ symmetry-breaking transition, it has been discussed in the connection to classical magnetic systems, unconventional superfluids, topological quantum information processing and storage, quantum phase transitions, spin-liquid, spin-ice phases, and high- $T_{c}$ superconductivity.

In this Rapid Communication, we propose to exploit the properties of polariton graphs to generate various frustrated states and spinor phases. In particular, we show that at the condensation threshold depending on the structure of the corresponding $X Y$ Hamiltonian the polariton condensates establish the relative phases that correspond to the classical ferromagnetic, antiferromagnetic, frustrated states, as well as the novel exotic states that can be associated with a spin wave, spin liquid, phase chaos, and crossbreeds between them.

The theoretical approach, presented here, is based on the well-known complex Ginzburg-Landau equation (cGLE) with a saturable nonlinearity $[26,27]$, that was successfully used for the modeling of polariton condensates. Written for the condensate wave-function $\psi$ in one dimension, the cGLE reads as

$$
\begin{aligned}
i \hbar \frac{\partial \psi}{\partial t}= & -\frac{\hbar^{2}}{2 m}\left(1-i \eta_{d} N_{R}\right) \psi_{x x}+U_{0}|\psi|^{2} \psi+\hbar g_{R} N_{R}(x) \psi \\
& +\frac{i \hbar}{2}\left(R_{R} N_{R}(x)-\gamma_{C}\right) \psi
\end{aligned}
$$

where $N_{R}=P(x) /\left(\gamma_{R}+R_{R}|\psi|^{2}\right)$ is the density of the hot exciton reservoir, $m$ is polariton effective mass, $U_{0}$ and $g_{R}$ are the strengths of effective polariton-polariton and polaritonexciton interactions, respectively, $\eta_{d}$ is the energy relaxation coefficient specifying the rate at which gain decreases with increasing energy, $R_{R}$ is the rate at which the reservoir excitons enter the condensate, $\gamma_{C}$ and $\gamma_{R}$ are the rates of the condensate polaritons and reservoir excitons' losses, respectively, and $P$ is the pumping into the reservoir. When pumped into several spots with the outflows from each spot reaching its neighbors the system establishes a global coherence with a chemical potential $\mu$ if the characteristics of the pump (intensity, spatial shape) and spots connectivity are not vastly different from one spot to another. The steady state of such a system satisfies

$$
\mu \Psi=-(1-i \eta n) \Psi_{x x}+|\Psi|^{2} \Psi+g n(x) \Psi+i[n(x)-\gamma] \Psi,
$$

$$
n=\frac{p(x)}{\left(1+b|\Psi|^{2}\right)},
$$

where we nondimensionalized Eq. (1) using $\psi \rightarrow$ $\sqrt{\hbar^{2} / 2 m U_{0} l^{2}} \Psi, \mathbf{r} \rightarrow l \mathbf{r}, t \rightarrow 2 m t l^{2} / \hbar$ and introducing the notations $g=2 g_{R} / R_{R}, \gamma=m \gamma_{C} l^{2} / \hbar, p=m l^{2} R_{R} P(\mathbf{r}) /$ $\hbar \gamma_{R}, \eta=\eta_{d} \hbar / m R_{R} l^{2}$, and $b=R_{R} \hbar^{2} / 2 m l^{2} \gamma_{R} U_{0}$. We choose the unit length as $l=1 \mu \mathrm{m}$. The Madelung transformation $\Psi=\sqrt{\rho} \exp [i S]$ relates the wave function to density $\rho=|\Psi|^{2}$ and velocity $u=S_{x}$. To derive the coupling strength we consider a single pumping spot centered at the origin and exponentially decaying to zero away from it. At large $x$, where $p(x)=0$, the velocity $u$ is given by the outflow wave-number $k_{c}=$ const with $\rho_{x} / \rho=-\gamma / k_{c}$, which can be integrated to yield $\rho \sim \exp \left[-x \gamma / k_{c}\right]$. From Eq. (2), therefore, we obtain $\mu=k_{c}^{2}-\gamma^{2} / 4 k_{c}^{2}$ at infinity.
The wave function, that approximately describes the system of $\ell$ identical pumping spots at positions $x_{i}$, takes the form $\Psi_{g}(x) \approx \sum_{i=1}^{\ell} \Psi_{i}\left(x-x_{i}\right)$ where the wave function of a single pumping spot centered at $x=x_{i}$ can be approximated by $\Psi_{i}\left(x-x_{i}\right)=\sqrt{\rho\left(x-x_{i}\right)} \exp \left[\left(i k_{c}\left|x-x_{i}\right|\right)+i \theta_{i}\right]$, where $\theta_{i}$ is a space-independent part of the phase. In the expression for the space varying phase we neglected the healing of the outflow velocity to zero at the center of the pump. This healing occurs on the length scale on the order of the width of the pump and can be neglected when evaluating the integral quantities over the entire sample. We note that nonlinearity of the system affects the assumption of the linear superposition of the individual wave functions in several ways: not only it rescales the single wave function in the superposition and decreases the chemical potential of the superposition in the steady state, but also it may bring about periodic and disordered fluctuations of the phase differences between the condensates for short distances between the pumping spots. However, for well-separated condensates this approximation is valid.

Depending on the pumping parameters, the system will lock with the relative phases $\theta_{i j} \equiv \theta_{i}-\theta_{j}$ between sites $i$ and $j$ to achieve the highest occupation number - the total amount of matter given by $\mathcal{D}=\int_{-\infty}^{\infty}\left|\Psi_{g}\right|^{2} d x$ [25]. To evaluate $\mathcal{D}$ we work in the Fourier space and write

$$
\begin{aligned}
\mathcal{D} & =\frac{1}{2 \pi} \int\left|\widehat{\Psi}_{g}(k)\right|^{2} d k \approx \frac{1}{2 \pi} \int\left|\sum_{i} \widehat{\Psi}_{i}(k)\right|^{2} d k \\
& =\ell \mathcal{D}_{0}+\frac{1}{\pi} \sum_{i<j} \int\left(\widehat{\Psi}_{i} \widehat{\Psi}_{j}^{*}+\text { c.c. }\right) d k,
\end{aligned}
$$

where $\mathcal{D}_{0}$ is the number of particles of a single isolated condensate, $\widehat{\Psi_{g}}(k)$ is the Fourier transform of $\Psi_{g}$, and

$$
\begin{aligned}
\widehat{\Psi}_{i}(k) & =\int_{-\infty}^{\infty} \Psi_{i}\left(x-x_{i}\right) \exp (-i k x) d x \\
& =\exp \left(-i k x_{i}\right) \int_{-\infty}^{\infty} \Psi_{i}(\alpha) \exp (-i k \alpha) d \alpha \\
& =\exp \left(-i k x_{i}+i \theta_{i}\right) \widehat{\psi}(k), \\
\widehat{\psi}(k) & =2 \int_{0}^{\infty} \sqrt{\rho(\alpha)} \exp \left(i k_{c} \alpha\right) \cos (k \alpha) d \alpha .
\end{aligned}
$$

Denoting the distances between the spots as $x_{i j}=x_{i}-x_{j}$ we substitute (5) into the integral in Eq. (4) to get

$$
\mathcal{D}=\ell \mathcal{D}_{0}+\frac{2}{\pi} \sum_{i<j} \cos \theta_{i j} \int_{0}^{\infty}|\widehat{\psi}(k)|^{2} \cos \left(k x_{i j}\right) d k .
$$

This implies that during the condensation the system of pumping spots establishes the phase difference in such a way as to minimize $\mathcal{H}_{X Y}$ where the coupling strengths are given by

$$
J_{i j}=\frac{2}{\pi} \int_{0}^{\infty}|\widehat{\psi}(k)|^{2} \cos \left(k x_{i j}\right) d k
$$

To obtain an analytical approximation of the coupling strengths and a criterion for the switching between the ferroand the antiferromagnetic connections along the chain of 
pumped condensate, we parametrize the amplitude of the condensate by the inverse width $\beta$ and the height $A: \sqrt{\rho(x)} \approx$ $A \exp [-\beta|x|]$. We expect the width and the height of the condensate to correlate with the width and the intensity of the pumping profile, respectively. For this approximation of the amplitude the integrals in Eqs. (7) and (8) can be evaluated exactly,

$$
\begin{gathered}
\widehat{\psi}(k)=2 A \int_{0}^{\infty} \exp \left(-\beta \alpha+i k_{c} \alpha\right) \cos (k \alpha) d \alpha \\
=\frac{2 A\left(\beta-i k_{c}\right)}{\beta^{2}+k^{2}-2 i \beta k_{c}-k_{c}^{2}}, \\
J_{i j}=\frac{8 A^{2}}{\pi} \int_{0}^{\infty} \frac{\left(\beta^{2}+k_{c}^{2}\right) \cos \left(k x_{i j}\right) d k}{\beta^{4}+\left(k^{2}-k_{c}^{2}\right)^{2}+2 \beta^{2}\left(k^{2}+k_{c}^{2}\right)} .
\end{gathered}
$$

Applying the residue theorem for evaluating the last integral, we obtain the closed-form expression for the coupling constants,

$$
J_{i j}=2 A^{2}\left[\frac{1}{\beta} \cos \left(k_{c} x_{i j}\right)+\frac{1}{k_{c}} \sin \left(k_{c} x_{i j}\right)\right] e^{-\beta x_{i j}} .
$$

This expression determines the switching of ferro- and antiferromagnetic couplings between the neighbors since the sign of $J_{i j}$ is set by the expression $\cos \left(k_{c} x_{i j}\right) / \beta+\sin \left(k_{c} x_{i j}\right) / k_{c}$. If the pumping profile is wide ( $\beta$ is small), the sign of the interactions is determined by $\cos \left(k_{c} x_{i j}\right)$, which is what we expect directly from Eq. (8) since $|\widehat{\psi}(k)|^{2} \sim \delta\left(k-k_{c}\right.$ ) for a wide pumping spot.

Now we consider a linear periodic chain of $\ell$ equidistant polariton condensates separated by $x_{i j}=d$. This chain can be achieved by creating a sequence of trapped condensates $[28,29]$ or by pumping condensates around a circle $[23,30]$. The corresponding $X Y$ model takes the form $\mathcal{H}=-J_{1} \sum_{i} \mathbf{s}_{i}$. $\mathbf{s}_{i+1}-J_{2} \sum_{i} \mathbf{s}_{i} \cdot \mathbf{s}_{i+2}$ where the sum is over all $\ell$ condensates with periodic boundary conditions. In the case of $J_{2}=0$ the model is integrable [31], whereas for $J_{2} \neq 0$ the exact solutions were found for a limited set of values of $J_{2} / J_{1}$. Frustrated phases of the classical as well as the quantum spin- $1 / 2$ system with nearest-neighbor and next-nearest-neighbor isotropic exchanges known as the Majumdar-Ghosh Hamiltonian have been extensively studied [32,33]. Classically, three regimes were identified for $\ell \rightarrow \infty$ : ferromagnetic for $J_{1}>0, J_{2}>$ $-J_{1} / 4$, antiferromagnetic for $J_{1}<0, J_{2}>J_{1} / 4$, and the frustrated (spiral) phase otherwise as the inset in Fig. 1 illustrates. In the frustrated phase the pitch angle of the spiral is $\phi=$ $\cos ^{-1}\left(-J_{1} / 4 J_{2}\right)$ [33]. Quantum fluctuations lower the ground state and shift the phase transition from the spin-liquid state at $J_{2}=0$ to a dimerized regime with a gap to the excited states at $J_{2}=0.2411 J_{1}$; the transition from the antiferromagnetic phase to dimerized singlets takes place at $J_{2}=J_{1} / 2$ [32]. In what follows, we elucidate if a polariton linear periodic chain is capable of reproducing the characteristics of classical regimes and what new physics arises due to nonlinear interactions of polaritons.

We plot the values of $J_{2} / J_{1}$ found from Eq. (11) in Fig. 1 and demonstrate that for the experimentally achievable polariton spot widths and wave vectors the values of $J_{2} / J_{1}$ from -0.3 to 0.3 should be realizable. This suggests that all three

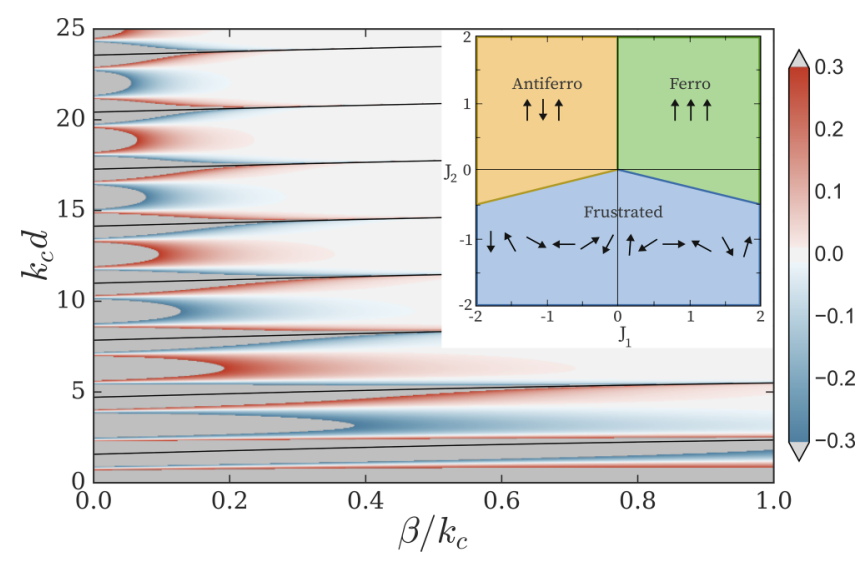

FIG. 1. A contour plot of frustration parameter $J_{2} / J_{1}$ as a function of $k_{c} d$ and $\beta / k_{c}$, where $k_{c}$ is the polariton outflow wave vector, $\beta$ is the inverse width of the polariton spot, and $d$ is the distance between the two adjacent spots. The coupling strengths $J_{1}$ and $J_{2}$ are calculated from Eq. (11) for the distances $d$ and $2 d$, respectively. The sign changes for $J_{1}$ are shown by the black solid lines. The inset shows the different regimes of the classical $X Y$ model on the $J_{2}-J_{1}$ plane: ferromagnetic, antiferromagnetic, and frustrated.

of the classical regimes, that are depicted in the inset of the Fig. 1, should be accessible in a linear periodic chain.

Next we consider 12 condensates in a linear periodic chain and numerically integrate Eq. (1) for a variety of distances between the spots. For each configuration we start from 100 random initial distributions of phases to find the ground-state configurations. Figure 2 identifies ferromagnetic, antiferromagnetic, and spiral spin-wave phases that represent the ground states of the one-dimensional $X Y$ model. The polariton densities (the blue solid lines) are clearly displaced from the

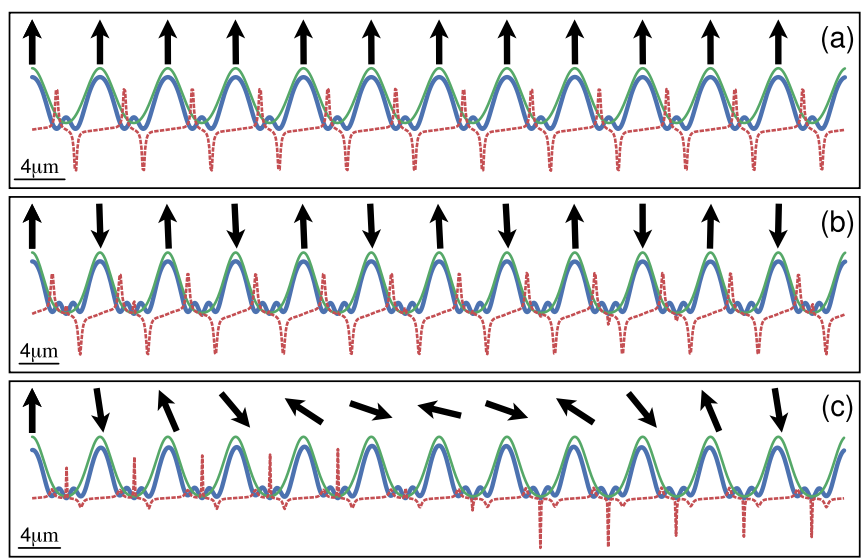

FIG. 2. Polariton densities (the blue solid line) and velocities (the red dashed lines) for 12 condensates obtained by numerical integration of Eq. (1) with periodical boundary conditions. Pumping profiles are shown with the green solid lines. Panel (a) shows the ferromagnetic state, (b) corresponds to antiferromagnetic state with a $\pi$-phase difference between the adjacent sites, and panel (c) shows the frustrated state. The distances between the nearest condensates are $d=5.4, d=6.9$, and $d=6.5 \mu \mathrm{m}$ for (a)-(c), respectively. The numerical parameters for one-dimensional simulations are $\eta=$ $0.4, b=1.5, \gamma=1, p_{0}=5, \sigma=0.4, g=2.5$. 

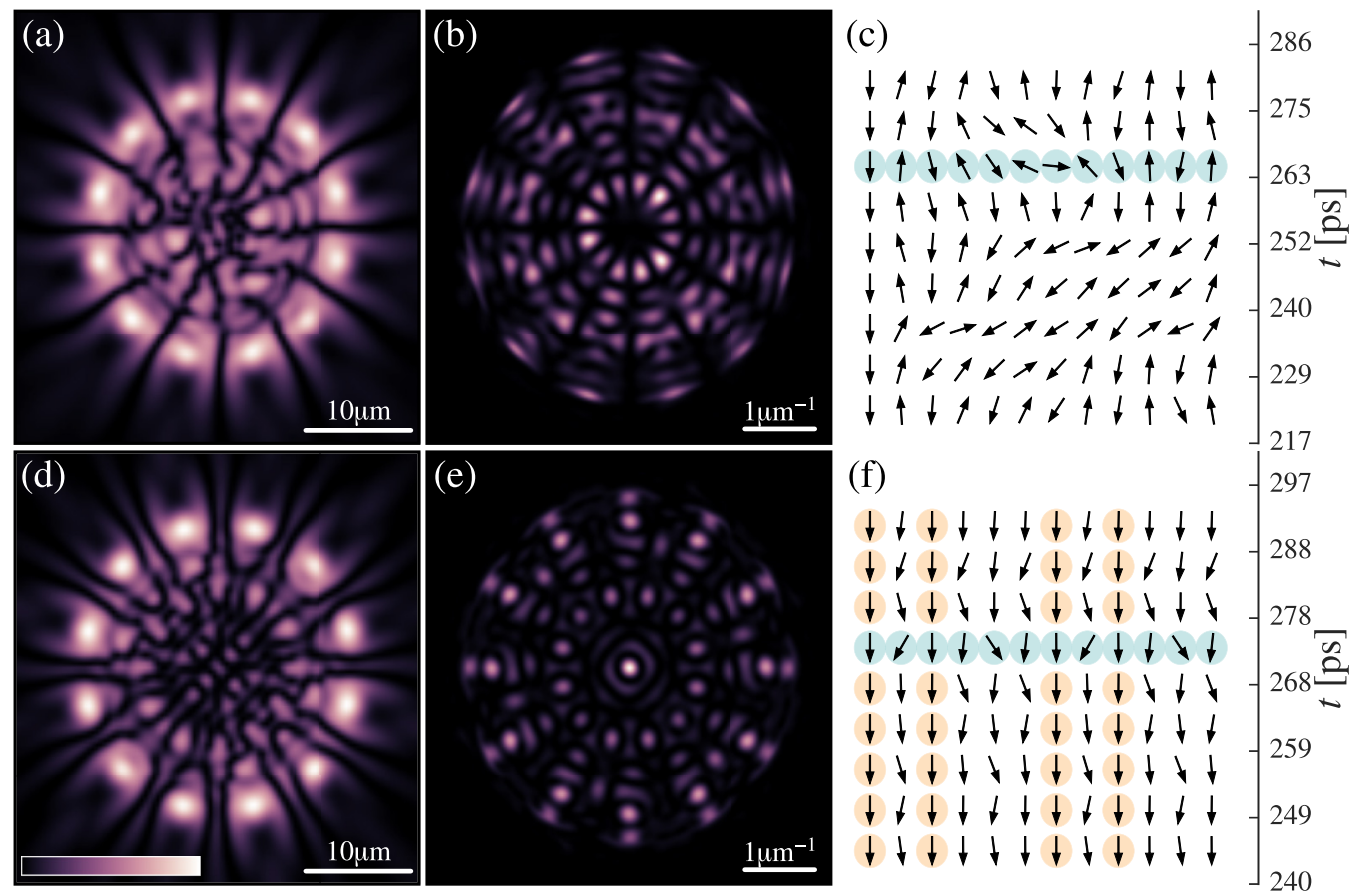

FIG. 3. Density snapshots (left panel) and the far-field emission (central panel) at a fixed time for 12 condensates arranged in a circle obtained by numerical integration of two-dimensional version of Eq. (1). The right panels show the evolution of the phases (relative to one fixed spin) between adjacent spots with (c) 12-ps and (f) 10-ps time laps between the spin configurations. The highlighted with blue circles spin configurations in (c) and (f) correspond to the density profiles (a) and (d) and the far-field emissions (b) and (e). The steady spins of the spots are marked with orange circles in (c) and (f). We use the simulation parameters that were found to agree with experimental data in our previous works $[23,30]$ except for the pumping intensity which is 2.6 times larger to bring about a nonstationary state; the distances between the adjacent sites are $6.4 \mu \mathrm{m}$ (a)-(c) and $7 \mu \mathrm{m}$ (d)-(f).

pumping profiles (the green solid lines) in the case of the spin-wave state which is depicted in Fig. 2(c). This state has a distinguished velocity pattern (the red dashed lines) compared to the other two classical states in Figs. 2(a) and 2(b). The experimental systems may suffer from noise, sample disorder, and interactions with impurities, so we have repeated the numerical simulations with the Langevin noise described in our previous work [25]. This noise introduces small oscillations around the steady states but has no effect on the time-averaged solutions that coincide with those found without the Langevin noise term.

We have also considered the full two-dimensional system with pumping spots equidistant around a circle. For pumping intensities just above the threshold the same classical phases are obtained. As the pumping intensity increases, the nonlinearity of the system destabilizes the frustrated state and produces spin fluctuations as Figs. 3(a)-3(c) illustrate, suggesting the formation of a nonstationary and chaotic spin wave which could be related to phase chaos [34]. Direct observation of nonstationary states in experiments would require time-resolved measurements on time scales that are challenging with current experimental configurations. Note that this is the lowest-energy state with respect to the $X Y$ Hamiltonian as it carries the largest number of particles for the given network configuration. It suggests that the pumping intensity can be used to continuously move between various state configurations: frustrated states, spin waves, spin liquids, phase chaos, etc., and to study transitions between them. For instance, Figs. 3(d)-3(f) demonstrate a nonstationary state of two spin waves of different periods (one or three spins). The spectral weights at a fixed time [Figs. 3(b) and 3(e)] reflect the symmetry of the lattice.

Nonstationary spin-wave states can be detected in the momentum- and energy-resolved photoluminescence spectra, which can be directly measured in the far field. Figure 4 shows the spectral weight $I(\omega, \mathbf{k})=\mid \iint \Psi(\mathbf{r}, t) \exp [-i \mathbf{k} \cdot \mathbf{r}-$ $i \omega t]\left.d t d \mathbf{r}\right|^{2}$ as a function of $\left(\omega, k_{x}, k_{y}=0\right)$. In the case of the nonstationary state depicted in Figs. 3(a)-3(c), spins reorient
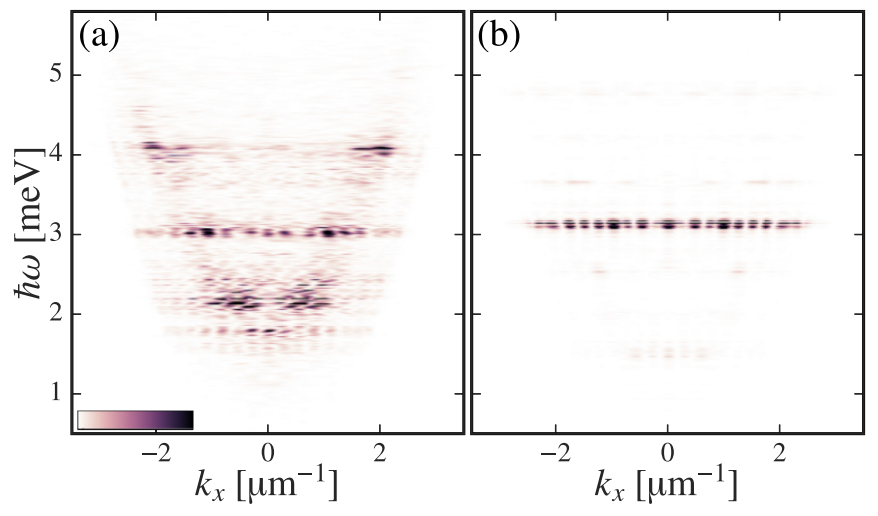

FIG. 4. The spectral weight plots for $k_{y}=0$ of the twodimensional nonstationary spin-wave states from Figs. 3(a)-3(c) in (a) and from Figs. 3(d)-3(f) in (b). Both plots are saturated at the same level of 0.2 to make the energy levels more visible and to provide an easier comparison between the states. 
themselves randomly with time cycling through different microstates with the density distribution depicted in Fig. 4(a). The state shown in Figs. 3(d)-3(f) is a more regular state with the energy spectrum shown in Fig. 4(b) indicating several well-separated energy levels. The stationary state would, in contrast, show only one energy level $[35,36]$.

The frustrated states that we found in the linear periodic chain of the polariton's graphs correspond to superfluids at nonzero quasimomentum and, therefore, exhibit nontrivial long-range phase order. The spiral phases spontaneously break time-reversal symmetry by generating bosonic currents around the sites of the polariton's graphs.

In conclusion, we considered polariton condensates arranged in a periodic linear chain. We evaluated the interaction strength between the condensates analytically in terms of the outflow wave vector, pumping width and strength, and the distance between the condensates. We have identified the parameter regime where the interactions beyond the next neighbors become important and lead to the appearance of the classical frustrated state. The nonlinear interactions beyond the linear approximation lift the ground-state degeneracy and facilitate the formation of a disordered state. We demonstrated that a sequence of polariton condensates is capable of supporting not only classical ground states of the $X Y$ model with nearest- and next-to-nearest-neighbor interactions, but also exotic states, such as nonstationary spin waves that may be associated with spin liquids or phase chaos. This observation opens a new path for studying such novel states of matter.

The authors acknowledge the support of the Skoltech NGP Program (Skoltech-MIT joint project). K.P.K. acknowledges the financial support from Cambridge Trust and EPSRC.
[1] S. Sachdev, Quantum magnetism and criticality, Nat. Phys. 4, 173 (2008).

[2] L. Balents, Spin liquids in frustrated magnets, Nature (London) 464, 199 (2010).

[3] F. H. L. Essler, H. Frahm, F. Gohmann, A. Klumper, and V. E. Korepin, The One-Dimensional Hubbard Model (Cambridge University Press, Cambridge, U.K., 2005).

[4] A. Auerbach, Interacting Electrons and Quantum Magnetism (Springer, New York, 1994).

[5] G. Misguich and C. Lhuillier, Frustrated Spin Systems, edited by H. T. Diep (World Scientific, Singapore, 2004).

[6] F. Alet, A. M. Walczak, and M. P. A. Fisher, Exotic quantum phases and phase transitions in correlated matter, Physica A 369, 122 (2006).

[7] P. W. Anderson, Resonating valence bonds: A new kind of insulator? Mater. Res. Bull. 8, 153 (1973).

[8] T.-H. Han, J. S. Helton, S. Chu, D. G. Nocera, J. A. RodriguezRivera, C. Broholm, and Y. S. Lee, Fractionalized excitations in the spin-liquid state of a kagome-lattice antiferromagnet, Nature (London) 492, 406 (2012).

[9] M. Lewenstein, A. Sanpera, V. Ahufinger, B. Damski, A. Sen, and U. Sen, Ultracold atomic gases in optical lattices: Mimicking condensed matter physics and beyond, Adv. Phys. 56, 243 (2007).

[10] M. Saffman, T. G. Walker, and K. Molmer, Quantum information with Rydberg atoms, Rev. Mod. Phys. 82, 2313 (2010).

[11] J. Struck, C. Ölschläger, R. Le Targat, P. Soltan-Panahi, A. Eckardt, M. Lewenstein, P. Windpassinger, and K. Sengstock, Quantum simulation of frustrated classical magnetism in triangular optical lattices, Science 333, 996 (2011).

[12] J. Simon, W. S. Bakr, R. Ma, M. E. Tai, Ph. M. Preiss, and M. Greiner, Quantum simulation of antiferromagnetic spin chains in an optical lattice, Nature (London) 472, 307 (2011).

[13] J. J. Pla, K. Y. Tan, J. P. Dehollain, W. H. Lim, J. JL Morton, F. A. Zwanenburg, D. N. Jamieson, A. S. Dzurak, and A. Morello, High-fidelity readout and control of a nuclear spin qubit in silicon, Nature (London) 496, 334 (2013).
[14] R. Hanson and D. D. Awschalom, Coherent manipulation of single spins in semiconductors, Nature (London) 453, 1043 (2008).

[15] M. Lemeshko, N. Y. Yao, A. V. Gorshkov, H. Weimer, S. D. Bennett, T. Momose, and S. Gopalakrishnan, Controllable quantum spin glasses with magnetic impurities embedded in quantum solids, Phys. Rev. B 88, 014426 (2013).

[16] T. E. Northup and R. Blatt, Quantum information transfer using photons, Nat. Photonics 8, 356 (2014).

[17] B. P. Lanyon, C. Hempel, D. Nigg, M. Müller, R. Gerritsma, F. Zähringer, P. Schindler et al., Universal digital quantum simulation with trapped ions, Science 334, 57 (2011).

[18] K. Kim, M-S. Chang, S. Korenblit, R. Islam, E. E. Edwards, J. K. Freericks, G-D. Lin, L-M. Duan, and C. Monroe, Quantum simulation of frustrated Ising spins with trapped ions, Nature (London) 465, 590 (2010).

[19] A. D. Corcoles et al., Demonstration of a quantum error detection code using a square lattice of four superconducting qubits, Nat. Commun. 6, 6979 (2015).

[20] S. Utsunomiya, K. Takata and Y. Yamamoto, Mapping of Ising models onto injection-locked laser systems, Opt. Express 19, 18091 (2011).

[21] A. Marandi, Z. Wang, K. Takata, R. L. Byer, and Y. Yamamoto, Network of time-multiplexed optical parametric oscillators as a coherent Ising machine, Nat. Photonics 8, 937 (2014).

[22] M. Nixon, E. Ronen, A. A. Friesem, and N. Davidson, Observing Geometric Frustration with Thousands of Coupled Lasers, Phys. Rev. Lett. 110, 184102 (2013).

[23] N. G. Berloff, M. Silva, K. Kalinin, A. Askitopoulos, J. D. Töpfer, P. Cilibrizzi, W. Langbein, and P. G. Lagoudakis, Realizing the classical XY Hamiltonian in polariton simulators, Nature Mater. 16, 1120 (2017).

[24] G. Tosi, G. Christmann, N. G. Berloff, P. Tsotsis, T. Gao, Z. Hatzopoulos, P. G. Savvidis, and J. J. Baumberg, Sculpting oscillators with light within a nonlinear quantum fluid, Nat. Phys. 8, 190 (2012).

[25] H. Ohadi, R. L. Gregory, T. Freegarde, Y. G. Rubo, A. V. Kavokin, N. G. Berloff, and P. G. Lagoudakis, Nontrivial Phase Coupling in Polariton Multiplets, Phys. Rev. X 6, 031032 (2016). 
[26] M. Wouters and I. Carusotto, Excitations in a Nonequilibrium Bose-Einstein Condensate of Exciton Polaritons, Phys. Rev. Lett. 99, 140402 (2007).

[27] J. Keeling and N. G. Berloff, Spontaneous Rotating Vortex Lattices in a Pumped Decaying Condensate, Phys. Rev. Lett. 100, 250401 (2008).

[28] P. Cristofolini, A. Dreismann, G. Christmann, G. Franchetti, N. G. Berloff, P. Tsotsis, Z. Hatzopoulos, P. G. Savvidis, J. J. Baumberg, Optical Superfluid Phase Transitions and Trapping of Polariton Condensates, Phys. Rev. Lett. 110, 186403 (2013).

[29] Y. Sun, P. Wen, Y. Yoon, G. Liu, M. Steger, L. N. Pfeiffer, K. West, D. W. Snoke, and K. A. Nelson, Erratum: BoseEinstein Condensation of Long-Lifetime Polaritons in Thermal Equilibrium, Phys. Rev. Lett. 118, 016602 (2017).

[30] K. Kalinin, M. Silva, J. D. Töpfer, W. Langbein, N. G. Berloff, and P. G. Lagoudakis, Giant vortices of controlled multiplicity in polariton lattices, arXiv:1710.03451.

[31] H. Bethe, Zur Theorie der Metalle. I. Eigenwerte und Eigenfunktionen der linearen Atomkette (On the theory of metals. I.
Eigenvalues and eigenfunctions of the linear atom chain), Z. Phys. 71, 205 (1931).

[32] C. K. Majumdar and D. K. Ghosh, On next-nearest-neighbor interaction in linear chain. I, J. Math Phys. 10, 1388 (1969); On next-nearest-neighbor interaction in linear chain. II, 10, 1399 (1969).

[33] R. Bursill, G. A. Gehring, D. J. J. Farnell, J. B. Parkinson, T. Xiang, and C. Zeng, Numerical and approximate analytical results for the frustrated spin-1/2 quantum spin chain, J. Phys.: Condens. Matter 7, 8605 (1995).

[34] O. V. Popovych, Y. L. Maistrenko, and P. A. Tass, Phase chaos in coupled oscillators, Phys. Rev. E 71, 065201(R) (2005).

[35] M. O. Borgh, J. Keeling and N. G. Berloff, Spatial pattern formation and polarization dynamics of a nonequilibrium spinor polariton condensate, Phys. Rev. B 81, 235302 (2010).

[36] M. O. Borgh, G. Franchetti, J. Keeling and N. G. Berloff, Robustness and observability of rotating vortex-lattices in an exciton-polariton condensate, Phys. Rev. B 86, 035307 (2012). 